\title{
OCHO NOTAS CRÍTICAS A DEMÓSTENES, PRIMERA FILÍPICA
}

\author{
Felipe Hernández Muñoz \\ Universidad Complutense
}

IV, 32, LIN. 15-18':

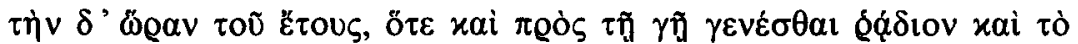

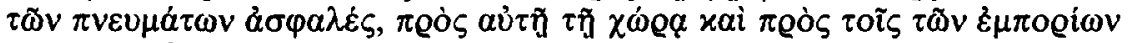

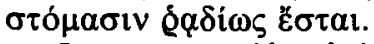

La construcción $\varepsilon i j \mu \mathfrak{i}+$ adverbio de modo ${ }^{2}$ se documenta:

a) Con nomen actionis como sujeto, tipo Heródoto IV, 134. 2:

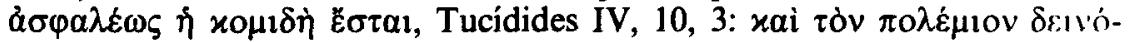

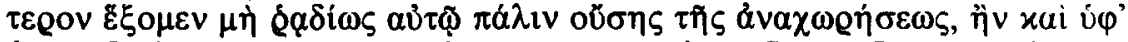
$\hat{\eta} \mu \tilde{\Phi} v \beta 1 \alpha \dot{\zeta} \eta \tau \tau l$ (pero, con algunos manuscritos, Stuart Jones corrige en

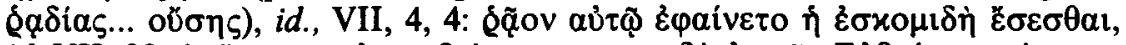

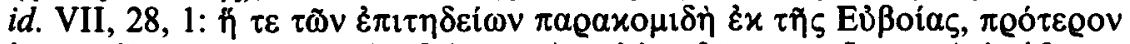

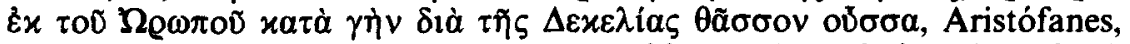

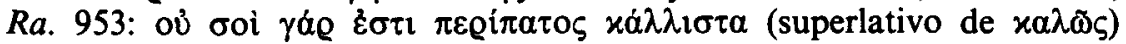

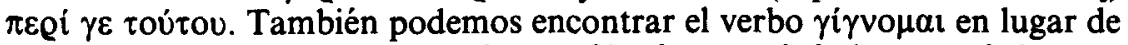

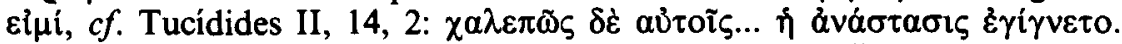
Las perifrasis son equivalentes al giro verbal correspondiente con xopi

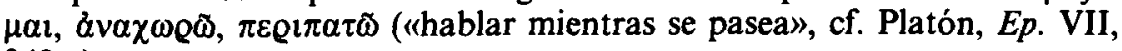
348 c), etc. 1980.

' Citamos por la edición de S. H. Butcher, Demosthenis Orationes, I, Oxford, 1903, reimp.

${ }^{2} \mathrm{Cf}$. Ch. H. Kahn, The verb «be» and its synonyms. Dordrecht-Boston, 1973, pp. 152-154. 
b) En giro impersonal (sc. «la situación, el curso de los acontecimien-

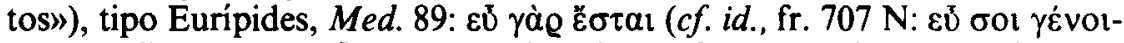

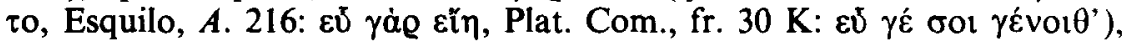

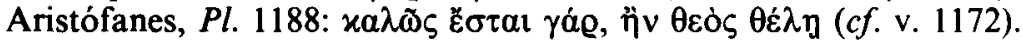

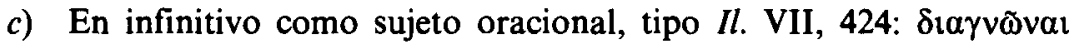

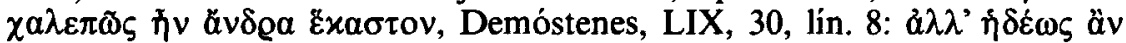

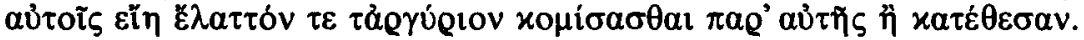

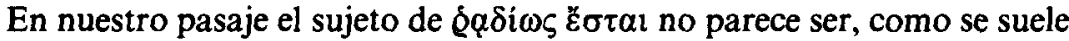

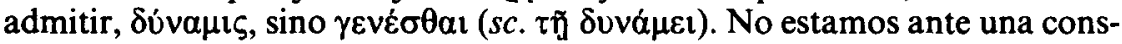
trucción del tipo a), sino $c$ ) (como en LIX, 30): «... el llegar a estar junto al propio territorio y las bocas de los puertos será fácil (para la fuerza).» En ese caso, debería suprimirse la "crux philologica" que algunos editores, como S. H. Butcher o M. Croiset, colocan en el pasaje.

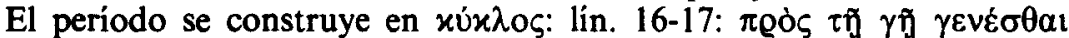

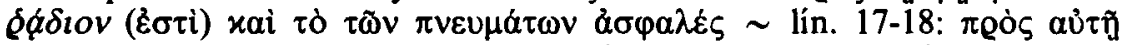

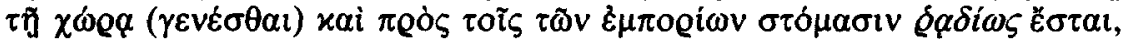
con la «variatio» de colocar los dos últimos miembros en quiasmo:

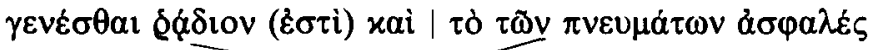

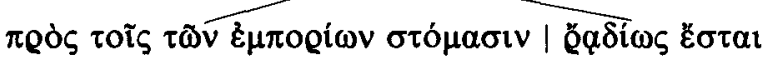

El xúx入os reitera una figura utilizada también en el período anterior:

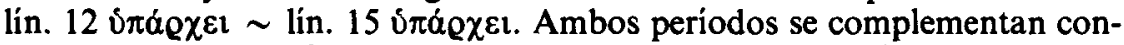
traponiendo $(\mu \dot{\varepsilon} v \ldots . . \delta \dot{\varepsilon})$ las actitudes sugeridas en la estación invernal y en la veraniega. Por no reparar en ello, se ha pensado que el segundo ítá $\chi \chi \varepsilon 1$ habría sustituido a algún otro verbo del original ${ }^{3} \mathrm{o}$ se ha querido justificar una trasposición del pasaje detrás del párrafo $44^{4}$. Parecidos problemas ha

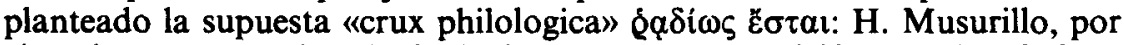
ejemplo, una vez desechada la innecesaria trasposición, corrige ¿q $\alpha i \omega \varsigma$ $\varepsilon \sigma \tau \alpha \imath$ en $\delta \varrho \mu \imath \sigma \theta \eta \dot{\sigma} \sigma \varepsilon \tau \alpha l ; M$. Croiset, sin embargo, cree más probable que haya caído algún verbo tras $\oint \alpha \delta i \omega \varsigma^{5}$.

\footnotetext{
3 Así. H. Richards («Notes on greek orators", $C R$ 1915, pp. 100-103), sin precisar a qué verbo.

'Como H. Musurillo, "A critical note on Demosthenes' First Philippic», CQ 51, 1957, p. 87.

s Démosthène Harangues, París, 1939, I, p. 44, nota ad. loc.: «verbum unum post ¿q̊ícos periisse videtur (fort. $\pi \circ \lambda \varepsilon \mu \varepsilon \tau v$ ) vel $\lambda \eta n \sigma \tau \varepsilon u ́ \varepsilon i v:$ nisi quid simile sub verbo $\delta \alpha \delta i \omega \varsigma$ latere existimemus»,
} 
Esta figura del $x u ́ x \lambda \circ \varsigma$ («anillo», «Ringkomposition») aparece con al-

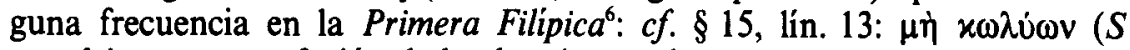
$x \alpha \tau \alpha \lambda \dot{\omega} \omega v$, por confusión de la abreviatura de $x \alpha \tau \alpha-$ con $x \omega$-, vista al revés por Weil al proponer $\left.x \alpha \tau \alpha x \omega \lambda \nu \omega^{\prime}\right)^{7}$, que recoge verbalmente $x \omega \lambda \tilde{\delta} \sigma \alpha 1 ; \S 16$,

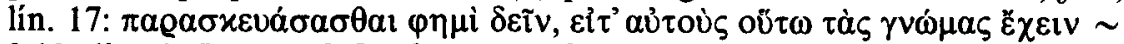

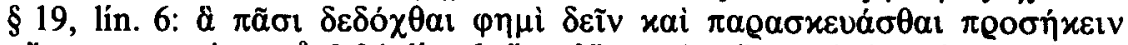

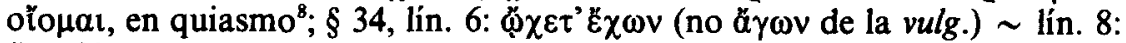
$\Phi \chi \varepsilon \varepsilon \tau^{\prime} \varepsilon^{\prime} \chi \omega v$.

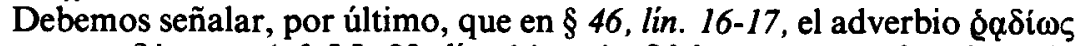

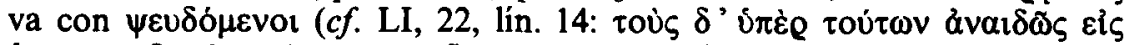

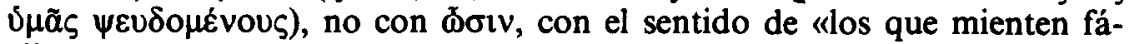
cilmente» («a la ligera»). El juego de cláusulas es -uu- -u- | -u-u, las dos más corrientes en Demóstenes:

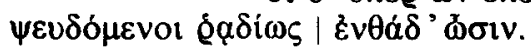

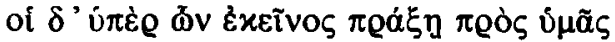

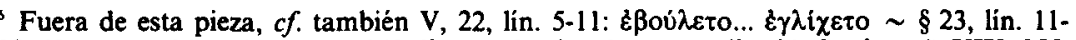

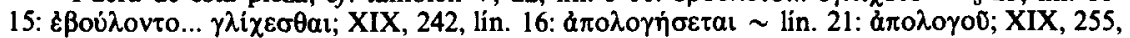

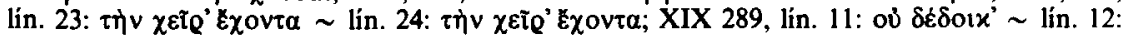

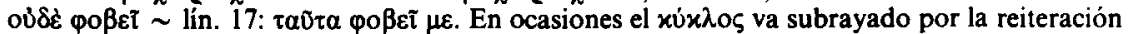
de los mismos preverbios, estilema recurrente en Demóstenes, como en XXII, 37: xata roũ-

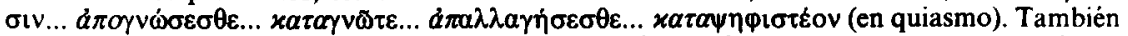

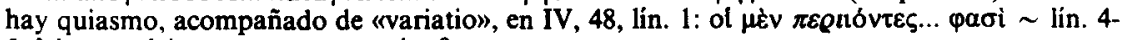

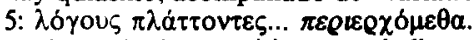

La "Ringkomposition" puede llegar, incluso, a estructurar piezas enteras (como es el caso del discurso Sobre la Corona, articulado en torno a la noción de Eరvvola: cf. XVIII, 1, lín. $7-$ $9 \sim \S 173$, lín. 20-21 $\sim 322$, lín. 17-18), o secciones importantes de ellas. Así ocurre con muchos de los esquemas de argumentación, normalmente en tres etapas: tesis general del orador, pruebas concretas que avalan dicha tesis y en las que debe fundamentarse la conclusión del auditorio, y recapitulación de la tesis inicial del orador, que debe ser asumida también por el auditorio (cf. XXII, 44-46, y 63-64; XXIII, 118-122).

${ }^{\prime} C f$. un caso análogo en Tucídides II, 64,2 : los manuscritos transmiten $x \omega \lambda v \theta$, pero Dobree, seguido por Herwerden, propone $x \alpha \operatorname{ta\lambda } \nu \theta \hat{n}\left(x^{2} \lambda \cup \theta \tilde{x}\right)$. M. Schmidt, por su parte, pre-

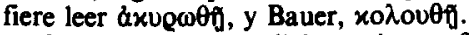

Este tipo de condicionamientos formales ha sido descuidado por B. Gaya Nuño en su excelente trabajo sobre estos sintagmas en Demóstenes (Sobre un giro de la lengua de Demós. tenes, Madrid, 1959). 


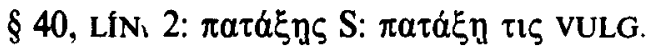

Aquí, como en XXI, 33, lín. 24-25, la lección de $S$ (de $\mathbf{S}^{1}$ en el pasaje citado en segundo lugar) es preferible, sin dejarnos llevar por una crítica textual de "conveniencia moral», como la practicada por Cobet y que, curiosamente, es aceptada por $\mathrm{J}$. Wackernagel ${ }^{9}$. La construcción con la llamada "segunda persona impersonal» es giro muy griego ( $c f$. ya en $\mathrm{Il}$. IV,

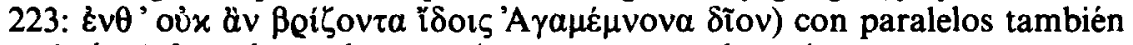
en latín (cf. crederes, diceres). Aunque se trate de acciones vergonzosas, en ambos casos la segunda persona es preferible como «lectio difficilior» frente al giro más trivial con el indefinido $\tau ו \varsigma$ y la tercera persona.

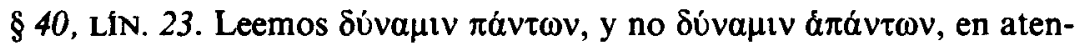
ción a que en Demóstenes $\tilde{\alpha} \pi \alpha \varsigma$ y $\pi \tilde{\alpha} \zeta$ alternan frecuentemente, pero, sobre

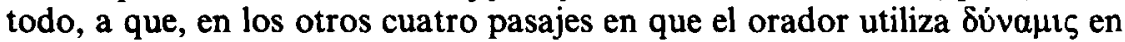
el discurso, evita la sucesión de tres breves, alargando la última ante dos

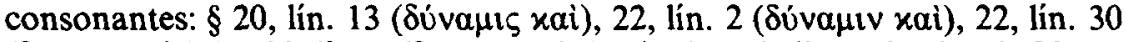

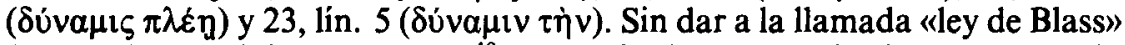
(matizada por Adams y Vogel) ${ }^{10}$ categoría de norma absoluta, parece, sin embargo, preferible la leve modificación del «textus receptus», aunque no lo hace así S. H. Butcher.

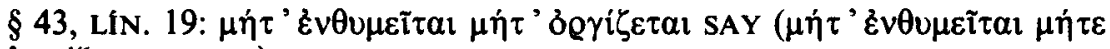

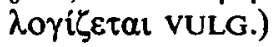

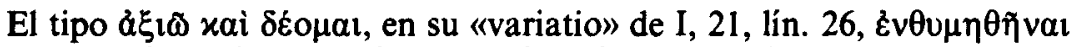

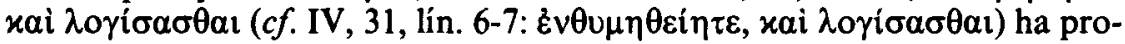
vocado, tal vez por "perseveratio", la «lectio facilior» de la vulgata, que da peor sentido. El copista se ha dejado llevar por la frase hecha y no ha

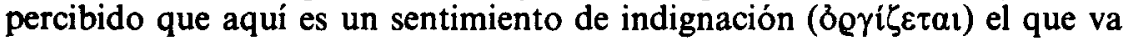

- Vorlesungen über Syntax, Basilea 1926-28², I, pp. 109-110.

${ }^{10}$ Cf. F. Blass, Die attische Beredsamkeit, III, Leipzig, 1983', reimp. Hildesheim-Nueva York, 1979, pp. 105 y 107, n. 5; D. Adams, «Demosthenes Avoidance of Breves», CPh 12, 1917 , p. 282; F. Vogel, «Die Kürzenmeidung in der griechischen Prosa des IV Jahrhunderts», Hermes 58, 1923, p. 88; F. Martín Garcia, Sobre las Simorias de Demóstenes, Ritmo, Estilo y Estructura, Ciudad Real, 1981, pp. 6-14. 
unido al de cálculo, para aludir, en expresión polar, al conjunto de las facultades intelectuales y afectivas del hombre ${ }^{11}$.

§ 43, Lín. 22-23. La trasposición de estas líneas, propuesta por Blass (y combinada con la de $\$ 37-38$, lín. 6-10, por $\mathrm{H}$. Musurillo) ${ }^{12}$, desconoce un uso muy demosténico ${ }^{13}$ de $\alpha \lambda \lambda \dot{\alpha} \mu \eta \dot{v} v$ para introducir la premisa menor, antes de la mayor, de un argumento: "Filipo no se detendrá» (premisa mayor); «el final de esta guerra es nuestra ruina» ( $=$ «si no se detiene, es nuestra ruina»; premisa menor que precede en lín. 20-22); conclusión (EI $\tau a$, con-

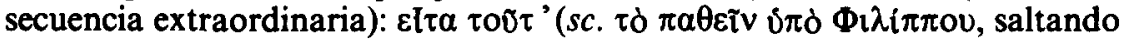

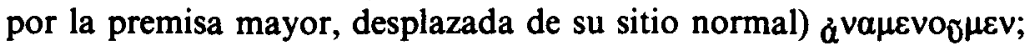

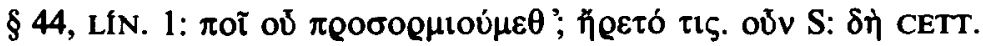
(TAMBIÉN HERMÓGENES Y [LONGINO])

Contra H. Musurillo, que se inclina por $\delta \dot{\eta}^{14}$, parece preferible ỡv ( $c f$. $\S 47$, lín. 20), que introduce una "conclusión de orden práctico" ${ }^{15}$ : el orador finge que alguien le pregunta no por la navegación en general, sino sobre un punto concreto en la costa donde atracar. A partir de la grafia $8 \mathbf{v} v$ se explica la confusión con $\delta \dot{n}$.

"El rechazo ateniense debe producirse en ambos planos, el intelectual y el afectivo-volitivo, pero, sobre todo, en un tercero: el de la acción. La clásica oposición $\gamma$ wón / dorń (funda-

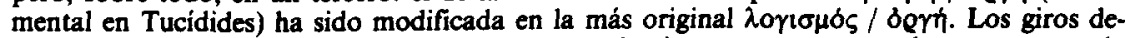
mosténicos de I, 21, lín. 26, y IV, 31, lin. 6-7, pueden interpretarse como pleonasmos, con la típica acumulación retórica, para aludir a la reflexión del auditorio, aunque también cabe

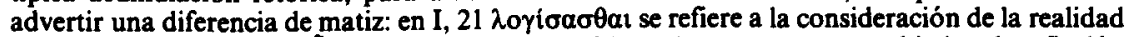

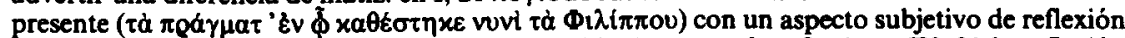

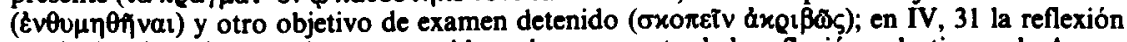
particular de cada ciudadano se considera el presupuesto de la reflexión colectiva en la Asam-

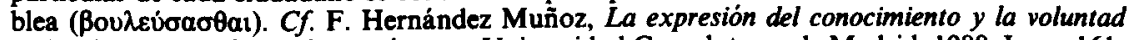
en los discursos politicos de Demóstenes, Universidad Complutense de Madrid, 1988, I, pp. 161, 184 y 192; II, pp. 497-498.

${ }_{12}$ Art. cit., p. 87. No obstante, después de varios ensayos al respecto, concluye por dejarlas en su sitio, en el que ya está en $P . O .15,1810$, del siglo II d. de J.C.

${ }_{13} C f$. J. D. Denniston, The Greek Particles, Oxford, $1954^{2}$. p. 346.

14 Loc. cit., of. J. D. Denniston, op. cit., p. 210.

is E. des Places, Etudes sur quelques particules de liaison chez Platon, Paris, 1929, p. 45. 


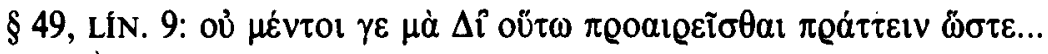

La trasposición de $\gamma \varepsilon$ (de valor enfático y limitativo) tras oũ $\tau \omega$, propuesta por Cobet y aceptada por S. H. Butcher, conforme al paralelo de

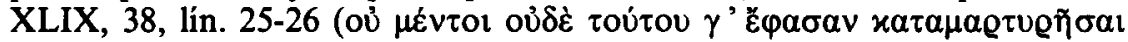
$\partial \nu \tau \dot{\alpha} \lambda \eta \theta \hat{\eta})$, pasa por alto que el énfasis recae aquí sobre oủ $\mu \dot{\varepsilon} v \tau o l$, como lo prueba el que $\mu \dot{\alpha} \Delta \dot{i} \alpha$ le siga, reforzando la negación. Cuando precede, sin embargo, se refiere al conjunto de la frase, $c f . \S 25$, lín. 24-25: $\mu \grave{a} \Delta \hat{\imath}$

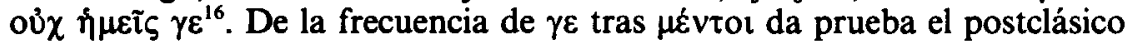

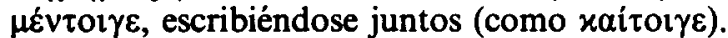

No quisiera cerrar estas notas sin mostrar públicamente mi agradecimiento al doctor Lasso de la Vega, quien amablemente las ha revisado antes de su publicación. Modestamente, a él quieren ir dedicadas, en agradecimiento a lo mucho que le debo como persona y como filólogo: $\lambda^{\lambda} \mu \varepsilon \gamma \alpha \dot{\lambda} \lambda \alpha$

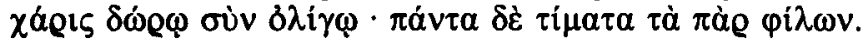

${ }^{16}$ Cf. G. Ronnet, Etude sur le style de Démosthène dans les discours politiques, Paris, 1951, reimp. 1971, p. 13. 